\title{
UNIVERSAL REGRESSIVE ISOLS
}

\author{
JOSEPH BARBACK
}

\begin{abstract}
E. Ellentuck introduced universal isols in Math. Z. 98 (1967), 1-8, to show how counterexamples in the arithmetic of the isols may be obtained in a uniform manner. Also Ellentuck was the first to prove, in unpublished notes, that there will be regressive isols that are universal. The present paper contains a relatively short proof that every infinite multiple-free regressive isol will be universal.
\end{abstract}

1. Preliminaries and basic ideas. We shall assume that the reader is familiar with the concepts and main results cited in the papers [1], [2] and [4]. We adopt the notation of [2]. The principal result proved in [2] is the following lemma.

LEMMA 1. Let $\alpha$ be a nonempty recursive set and let $f$ be any increasing recursive function $\mathrm{w}$ hose range is $\alpha$. Let $\alpha_{12}$ denote the set of regressive isols that belong to the extension of $\alpha$ to the isols, and let $D_{f}$ denote the canonical extension of $f$ to the isols. Then $\alpha_{R}=D_{f}\left(\Lambda_{R}\right)$.

The basic idea for the proof of the main theorem in this paper arises from observing an elementary yet useful way of characterizing $\%$ and $\alpha_{R}$ for particular recursive sets $\alpha$. This way is given in the following lemma and theorem. The techniques employed in the proof of the theorem are similar to those in [1] and [2].

LEMMA 2. Let $x$ be an infinite recursive set of numbers and let $f$ denote the principal function of $\alpha$. Then there will be functions $g$ and $h$ such that

(1) $g$ and $h$ are each increasing and recursive,

(2) $g$ ranges over an infinite set, and

(3) $f(x)=2 \cdot g(x)+h(x)$, for each number $x$, if and only if the complement of $\alpha$ is also an infinite set.

Proof. Assume first that there are functions $g$ and $h$ having the properties (1)-(3). Let us also assume that the complement of $\alpha$ is a finite set. Then there would be a number $k$ such that

$$
f(k)=y, \quad f(k+1)=y+1, \quad f(k+2)=y+2, \cdots .
$$

Received by the editors April 17, 1972.

AMS (MOS) subject classifications (1970). Primary 02F40.

Key words and phrases. Universal isol, regressive isol, multiple-free isol.

(C) American Mathematical Society 1973 
Since both $g$ and $h$ are increasing functions, it would then follow from property (3), that

$$
g(k+1)=g(k+2)=g(k+3)=\cdots .
$$

Therefore $g$ would have a finite range, and this would contradict property (2). Therefore it must be that the complement of $\alpha$ is infinite.

Let us assume now that both $\alpha$ and its complement are infinite sets. Define functions $g$ and $h$ in the following way. Let $g(0)=0$ and let $h(0)=$ $f(0)$. Assume that the values for $g$ and $h$ have been defined for values up to and including the number $y$. To define the functional values at $y+1$, we consider two cases. Let $a=f(y)$ and let $b=f(y+1)$.

Case 1. $b=a+1$. Define

$$
g(y+1)=g(y), \text { and } h(y+1)=1+h(y) .
$$

Case 2. $b=a+u+2$ with $u \geqq 0$. Define

$$
g(y+1)=1+g(y), \text { and } h(y+1)=u+h(y) .
$$

Because $\alpha$ is an infinite recursive set and $f$ is its principal function, $f$ will be a strictly increasing recursive function. Combining this property with the definitions above, it is easy to see that properties (1) and (3) will be true. Also, because the complement of $\alpha$ is an infinite set, Case 2 above will occur infinitely often, and this will mean that $g$ will range over an infinite set. This gives property (2), and also proves the lemma.

THEOREM. Let $\alpha$ be an infinite recursive set and let $Y$ be an infinite regressive isol belonging to $\alpha_{12}$. If the complement of $\alpha$ is also an infinite set, then there will be regressive isols $S$ and $T$ with $S$ infinite and $Y=2 S+T$.

Proof. Let $f$ denote the recursive principal function of $\alpha$. Assume that the complement of $\alpha$ is an infinite set and let $g$ and $h$ be functions chosen to have the properties as in Lemma 2. By Lemma 1 we know that $\alpha_{R}=D_{f}\left(\Lambda_{R}\right)$. Also, by combining property (3) in Lemma 2 with the well-known metatheorem of A. Nerode for such statements, it follows that $D_{f}(C)=$ $2 D_{g}(C)+D_{l}(C)$, for all isols $C$. Because $Y$ is in $\alpha_{R}$ then $Y=D_{f}(U)$ for some regressive isol $U$. Therefore, also $Y=D_{f}(U)=2 D_{g}(U)+D_{h}(U)$.

Since $Y$ is infinite $U$ will be also, and because $g$ and $h$ are each increasing and recursive functions $D_{g}(U)$ and $D_{h}(U)$ will each be regressive isols. Finally, we would like to note that $D_{g}(U)$ will be an infinite isol. This property may be verified in the following way. Consider the value of $D_{g}(U)$ expressed as an infinite series of isols, as given in [1, Proposition 2], and observe that in this series the associated $e$-difference function of $g$ will be positive infinitely often since the range of $g$ is an infinite set. Combining this form of an infinite series representation of $D_{g}(U)$ with the property that 
$U$ is an infinite regressive isol, it is easy to verify that the value of $D_{g}(U)$ will be infinite. If we set $S=D_{g}(U)$ and $T=D_{h}(U)$ then the desired result of the theorem will follow.

2. Universal and multiple-free isols. In view of $[5$, p. 4], a universal regressive isol may be defined in the following way: A regressive isol $U$ is universal if, for every recursive set $\alpha, U \in \alpha_{R}$ implies the complement of $\alpha$ is a finite set. An infinite isol $Y$ is called multiple-free, if for every isol $B$, $2 B \leqq Y$ implies $B$ is a finite isol. Multiple-free isols were introduced and studied in [4]. An example of an infinite regressive isol that is multiple-free appears in [3]. We can obtain directly from the theorem in $\S 1$ the following result, and it is the main theorem of the paper.

THEOREM. Every infinite multiple-free regressive isol is universal.

M. Hassett has shown that there will be universal regressive isols that are not multiple-free (not yet published). From this result we see that the converse of the previous theorem will not be true.

\section{REFERENCES}

1. J. Barback, Recursive functions and regressive isols, Math. Scand. 15 (1964), 29-42. MR 31 \#1189.

2. $\longrightarrow$, On recursive sets and regressive isols, Michigan Math. J. 15 (1968), 27-32. MR 37 \#64.

3. - Two notes on recursive functions and regressive isols, Trans. Amer. Math. Soc. 144 (1969), 77-94. MR 41 \#6691.

4. J. C. E. Dekker and J. Myhill, Recursive equivalence types, Univ. California Publ. Math. 3 (1960), 67-213. MR 22 \#7938.

5. E. Ellentuck, Universal isols, Math. Z. 98 (1967), 1-8. MR 35 \#5315.

Department of Mathematics, State University College of New York, Buffalo, NEW YORK 14222 